\title{
Buenas Prácticas Docentes Para Profesor De Inglés En El Ámbito Universitario
}

\author{
Daniela Fernanda Guano Merino \\ Magister en Lingüística Aplicada a la Enseñanza Bilingue Español - Inglés \\ Docente de la Escuela Superior Politécnica de Chimborazo, \\ Centro de Idiomas, Ecuador \\ Marcelo Eduardo Allauca Peñafiel \\ Magister en Interconectividad de Redes \\ Técnico Docente de la Escuela Superior Politécnica de Chimborazo, \\ Centro de Idioma, Ecuador \\ Salazar Silva Verónica Maribel \\ Licenciada en Ciencias de la Educación, Profesora de Idiomas Ingles \\ Técnico Docente de la Escuela Superior Politécnica de Chimborazo, \\ Centro de Idioma, Ecuador
}

Doi:10.19044/esj.2018.v14n20p25 URL:http://dx.doi.org/10.19044/esj.2018.v14n20p25

\begin{abstract}
The following investigation's objective was to establish that virtual platforms are considered as didactic support for good teaching practices in English language teaching. The research is quasi-experimental of the transversal type that makes a bibliographic, statistical and descriptive analysis. It considered two groups control and experimental; which were represented by 94 students who entered the first level of the Language Center of the Escuela Superior Politecnica de Chimborazo. The members of each group were evaluated before and after developing the academic activities, through a set of activities established in an evaluation rubric, which were validated by academic experts in the English area. The experimental group used a virtual platform; while the experimental group applied the traditional processes (without virtual platform). Both groups developed the contents of a micro curricular plan with an evaluation rubric. Through the scientific and hypothetical method, the evaluations obtained in a partial and final manner were compared; determining that, the academic performance of the experimental group improved with respect to the traditional one, concluding that the use of the virtual platforms it is a good teaching practice for the teaching of the English language.
\end{abstract}


Keywords: Good Teaching Practices, English, University Area, Virtual Platform.

\section{Resumen}

El objetivo de la presente investigación, fue establecer que las plataformas virtuales son consideradas como apoyo didáctico para las buenas prácticas docentes en la enseñanza del idioma inglés. La investigación es cuasi-experimental de tipo transversal que hace un análisis bibliográfico, estadístico y descriptivo. Se consideró dos grupos el de control y experimental; que estuvieron representados por 94 estudiantes que ingresaron al primer nivel del Centro de Idiomas de la Escuela Superior Politécnica de Chimborazo. Los integrantes de cada grupo fueron evaluados antes y después de desarrollar las actividades académicas mediante un conjunto de actividades, establecidos en una rúbrica de evaluación; que fueron validadas por expertos académicos en el área de inglés. El grupo experimental usó una plataforma virtual; mientras que el grupo de control, aplicó los procesos de manera tradicionales (sin plataforma virtual). Ambos grupos desarrollaron los contenidos de un plan micro curricular con una rúbrica de evaluación. Mediante el método científico e hipotético, se compararon las evaluaciones obtenidas de manera parcial y final; determinando que, el rendimiento académico del grupo experimental mejoró con respecto al tradicional, concluyendo que el uso de una plataforma virtual es una buena práctica docente para la enseñanza del idioma inglés.

Palabras claves: Buenas Prácticas Docentes, Inglés, Ámbito Universitario, Plataforma Virtual.

\section{INTRODUCCIÓN}

Las buenas prácticas en el ámbito educativo, representan el conjunto de intervenciones que el docente aplica durante el proceso de enseñanzaaprendizaje, con el propósito de maximizar los resultados positivos; privilegiando la formación integral de estudiante, minimizando el fracaso y la deserción escolar (Fingermann, 2013).

Para Vidal y Morales (2009) las buenas practicas, es un concepto que está asociado a procesos de calidad docente y "a las intervenciones educativas que facilitan el desarrollo de actividades de aprendizaje, en las que se logra con mayor eficiencia los objetivos formativos previstos; además de otros aprendizaje de alto valor educativo". Por tanto, las buenas prácticas docentes se convierten en acciones destacables e imitables que poseen en sí misma un valor añadido como tal.

En los Estados Unidos, las buenas prácticas son entendidas como "aquello que funciona", es decir, que logra lo que se espera y que resulta bien. La literatura relacionada con las buenas prácticas docentes la conceptualizan 
como una oportunidad de desarrollar un conjunto de habilidades y la adquisición de conocimientos que permita al estudiante mejorar su calidad de vida (UNESCO, 2014).

(Chaves, 2016; Gabaldón, 2011; Yáñez \& Soria, 2017; Osorio, 2017) además de resaltar su contribución a mejorar la calidad de aprendizajes, todos coinciden en señalarla como una práctica innovadora que ha sido evaluada, probada y que ofrece buenos resultados.

Por otro lado, se destaca que no todo planteamiento innovador es sinónimo de buena práctica; a pesar de que una experiencia innovadora puede ser una buena práctica, no siempre una práctica es innovadora. La innovación y la buena práctica deben ir estrechamente relacionadas no obstante, los criterios para establecer si una experiencia es innovadora o si una experiencia es una práctica son totalmente diferentes (Rosenfeld \& Fernández, 2012).

La buena práctica posee en sí mismo, un valor añadido que le da una connotación como tal y se relaciona con ciertos criterios que permiten reconocer, que la buena práctica puede ser adaptada a las necesidades y pertinencias del ámbito donde se la requiera. Consecuentemente se la orienta en dos ámbitos: en lo que el profesor debe realizar para que la actividad sea considerada una buena práctica y en los resultados que se puede tener en los estudiantes (Junta de Andalucía, 2012).

Fingermann (2013), dentro de las intervenciones de buenas prácticas docentes se deben considerar una planificación racional de las clases. Atendiendo las necesidades y características de los estudiantes, los recursos materiales disponibles y el tipo establecido; conjuntamente a ello y a la par, deberá existir una evaluación constante de los procesos y resultados, a través de la implementación de estrategias de motivación y de enseñanza.

A todo ello, es necesario guiar el aprendizaje de contenidos conceptuales, procedimentales y actitudinales a fin de incentivar el trabajo grupal cooperativo promoviendo el aprendizaje significativo, implementando las nuevas tecnologías.

Rubio (2010), Existen ciertos factores que facilitan la realización de las buenas prácticas docentes entre las que se encuentran:

- Factores relacionados a los estudiantes: se refiere al grado de homogeneidad, características, intereses y conocimientos previos.

- Factores relacionados con los docentes: en el que se considera la habilidad para el uso de las TIC.

- Factores relacionados con el centro docente: en el que se toma en cuenta la infraestructura física, aulas escolares equipadas y con acceso a internet, biblioteca-mediateca en funcionamiento, aulas de informática suficientes, disponibilidad de pizarra electrónica, entre otros (Marqués, 2002). 
- Factores relacionados con la administración educativa: se refiere a las buenas actuaciones de la administración educativa que involucren los planes de formación, apoyo docente y seguimiento de las experiencias que se realizan; además de la disponibilidad de un asesoramiento a través de especialistas, temáticos, técnicos.

Dentro de los factores relacionados con los docentes, se considera el uso de las TIC entre ellas las Plataformas virtuales educativas, que son sitios web de aprendizaje que permite al profesor contar con un espacio virtual en internet, donde se ubica información acorde a determinado conocimiento que se desea impartir, enlazando recursos digitales multimedia para desarrollar test, promover debates, comunicación, obtener una estadística de evaluación y uso; y demás herramientas que se crea necesario incluir para facilitar la experiencia de aprendizaje.

Las plataformas virtuales educativas contribuyen a mejorar los procesos de enseñanza, pues incorporan nuevas formas de interacción docente-estudiante, dinamizan la educación, $\mathrm{Su}$ adaptabilidad a las necesidades y requerimientos de los estudiantes y docentes de un Centro de Idiomas Universitario, son consideradas como una herramientas didácticas que ayudan en la práctica activa de evaluación y retroalimentación de temas que se desarrollan en clase.

Según VIU (2015), entre los múltiples beneficios que proporcionan las plataformas virtuales se consideran las siguientes:

- Centralización y automatización de la gestión del aprendizaje.

- Flexibilidad para ser adaptada tanto a los planes de estudio como a los contenidos y estilo pedagógico de la institución educativa.

- Interactividad porque convierte al participante en protagonista de su propio aprendizaje a través de los servicios guiados.

- Estandarización es una característica que permite utilizar cursos realizados por terceros, personalizando el contenido y reutilizando el conocimiento.

- Escalabilidad para funcionar con una cantidad variable de usuarios según las necesidades educativas.

- Funcionalidad según los requerimientos y necesidades de los usuarios.

- Usabilidad para utilizar la plataforma con facilidad a fin de contribuir a alcanzar un determinado objetivo.

- Ubicuidad es la capacidad que tiene la plataforma para generar tranquilidad al usuario y provocarle la certeza de que todo lo que necesita lo va a encontrar en dicho entorno virtual.

- Integración con otras aplicaciones que le permiten medir el impacto, eficacia y sobe todo el coste de las actividades de formación. 
Además de todos estos beneficios se debe indicar el alto nivel de motivación que despiertan, puesto que crean ambientes de aprendizaje personalizados y estimulan en el estudiante el uso de las nuevas tecnológicas conjuntamente con su capacitación.

En el ámbito universitario, el manejo del inglés como lengua extranjera es una herramienta que brinda oportunidades de progreso dentro de una sociedad globalizada y competitiva. Los estudiantes que acceden a la educación universitaria de reciente ingreso deben aprobar seis niveles del idioma extranjero como requisito curricular, cuyo propósito es dotar a los estudiantes del conocimiento necesario para el dominio de las cuatro habilidades comunicativas (escuchar, leer, escribir y hablar).

En base a una encuesta realizada por los autores a los estudiantes del contexto de la presente investigación, se pudo observar que el $40 \%$ de estudiantes tienen un bajo nivel de conocimientos del idioma extranjero, deficientes bases para el desarrollo de los procesos de enseñanza aprendizaje, lo cual se refleja en el rendimiento académico y deserción escolar.

En el contexto de la investigación, la enseñanza del idioma inglés se venía realizando de forma tradicional, sin el uso de una plataforma virtual como herramientas didáctica para el desarrollo de los contenidos que constan en el sílabo.

Los sílabos tienen diversas formas de evaluación (libertad de catedra), para medir el nivel de conocimiento adquirido por el estudiante. Razón por la cual, a los docentes se les motivo para el uso de herramientas tecnológicas como son las plataformas virtuales a fin de despertar interés, facilite el aprendizaje del idioma inglés y conocer si se mejoró el conocimiento y el rendimiento académico de los estudiantes universitarios.

\section{METODOLOGÍA}

La investigación fue cuantitativa; cualitativa ya que a través del análisis bibliográfico se obtuvo información sobre las buenas prácticas docentes como herramienta didáctica; y cuantitativa ya que se realizó una medición y análisis pre y post evaluatorio de los conocimientos de los estudiantes del Nivel 1 de un Centro de Idiomas de la Escuela Superior Politécnica de Chimborazo, a través de la medición de frecuencia y porcentajes; utilizando métodos estadísticos de medición.

El diseño de la investigación fue cuasi-experimental de tipo transversal, ya que existieron dos momentos temporales distintos en dos grupos diferentes (PRE Y POST). La muestra está representada por dos grupos que ya estaban formados antes del experimento; por tanto los estudiantes no se asignaron al azar, ni fueron emparejados (Hernández, 2015).

El grupo 1 denominado de control, representa el conjunto de estudiantes del primer nivel paralelo "A", que desarrollaron sus actividades 
académicas de forma tradicionalmente utilizando el sílabo. Las evaluaciones obtenidas (calificaciones) representan el rendimiento académico, las que sirvieron para promediar el conocimiento alcanzado en el aprendizaje del idioma Inglés.

El grupo 2 denominado experimental, representa el conjunto de estudiantes del primer nivel paralelo "A", que utilizaron un texto básico con los contenidos del sílabo y el apoyo didáctico de una plataforma virtual educativa como buena práctica docente. Mediante la plataforma virtual, el docente y los estudiantes interactuaron para realizar las tareas que fueron evaluadas para obtener la información referente al rendimiento académico alcanzado.

El semestre universitario consta de tres parciales con una duración de seis meses, una prueba diagnóstica inicial a clases y el promedio o rendimiento final logrado por los estudiantes al finalizar el nivel académico. Se tomó en cuenta, que al Centro de Idiomas acuden los estudiantes de diferentes instituciones educativas públicas y privadas así como de diferentes sectores geográficos del país.

\section{Población y muestra}

\begin{tabular}{|l|l|l|l|}
\multicolumn{5}{|c}{ Tabla 1: Población } \\
\begin{tabular}{|l|l|l|l|}
\hline GRUPOS & fr & FA \\
\hline Grupo 1 & Control & 47 & $50 \%$ \\
\hline Grupo 2 & Experimental & 47 & $50 \%$ \\
\hline & TOTAL & $\mathbf{9 4}$ & $\mathbf{1 0 0 \%}$ \\
\cline { 2 - 4 }
\end{tabular}
\end{tabular}

Fuente: Centro de Idiomas ESPOCH

Elaborado por: Autores

La técnica utilizada para la investigación fue la encuesta y mediante un conjunto de actividades se evaluaron y se obtuvieron los resultados. Del total las calificaciones se obtuvieron los promedios parciales y finales. El análisis descriptivo de los resultados se realizó mediante el software estadístico SPSS v21 que permitió establecer diferencias significativas entre los grupos.

Las rubricas de evaluación para los dos grupos fueron las mismas, y estuvieron revisadas por pares académicos.

Para alcanzar la meta de la investigación en primera instancia, la comisión de carrera conformada por docentes y expertos académicos del área, revisaron los contenidos de la estructura de los sílabos incluida la evaluación, a fin de garantizar que las actividades académicas que se desarrollen en los dos grupos sean similares.

Se consideró al inicio de las actividades académicas una evaluación previa a fin de establecer el conocimiento que tienen los estudiantes que 
ingresan a la educación universitaria con respecto a los contenidos del sílabo a tratar.

En un periodo de 2 semanas se realizó un programa de capacitación, donde se instruyó a los estudiantes y docente sobre el uso de la plataforma virtual, para que sea un apoyo a las actividades académicas. Mediante los planes de clases se monitorearon las actividades y se verificaron que las evaluaciones planificadas se vayan ejecutando en los plazos establecidos, las mismas que se registraron en un sistema de académico institucional.

Una vez obtenidas las calificaciones fueron analizadas e interpretadas y sometidas a una prueba estadística T-student que permitió conocer el rendimiento alcanzado por cada grupo para compáralo y determinar las conclusiones pertinentes a la investigación.

\section{RESULTADOS}

Tabla 2. Conocimiento general previo

\begin{tabular}{llll}
\hline Conocimiento & $\square$ & S & N \\
\hline Diagnóstico Control & 4,22 & 1,86 & 47 \\
Diagnóstico Experimental & 3,37 & 1,64 & 47 \\
\hline
\end{tabular}

Fuente: Registro de calificaciones

Elaborado por: Autores

La tabla 2 muestra los resultados del análisis descriptivo del diagnóstico realizado a los estudiantes que ingresan a la universidad y reciben la asignatura de inglés. Todos se ubican en el Nivel 1 y fueron distribuidos en dos paralelos homogéneos e iguales en número. El promedio para el grupo de control es de 4,22 $\pm 1,86$ en un número de 47; mientras que el grupo experimental tiene un promedio inicial de 3,37 $\pm 1,64$ en un grupo de 47 .

El diagnóstico tiene el objetivo de determinar el nivel de conocimientos del estudiante a partir del cual, el docente impartirá clases, de acuerdo a los resultados obtenidos se observa que los estudiantes tienen un nivel deficiente. Además se determinó que el grupo experimental alcanza un promedio inicial de 3,37; es decir 0,87 más bajo con respecto al grupo de control.

Los resultados corroboraron los resultados de los exámenes internacionales y estandarizados aplicados (TOEFL), donde se indica que los estudiantes del último año de Bachillerato General Unificado del Ecuador de los planteles educativos públicos se ubican en un nivel bajo (Heredia, 2017; Ortega, 2017).

Tabla 3: Notas Primer Parcial

\begin{tabular}{lcll}
\hline Primer parcial & $\square$ & s & N \\
\hline Grupo Control & 7,29 & 0,44 & 47 \\
Grupo Experimental & 7,12 & 0,68 & 47 \\
\hline
\end{tabular}

Fuente: Plataforma de calificaciones OASIS

Elaborado por: Autores 
El análisis descriptivo de la tabla 3, señalo que los participantes del grupo control tienen un promedio del primer parcial de 7,29 $\pm 0,44$ utilizando el método tradicional; mientras que, el grupo experimental obtuvo 7,12 $\pm 0,68$ con el uso de un texto y la plataforma virtual educativa como herramienta didáctica.

Los resultados señalan un mejor promedio de los estudiantes del grupo de control quienes acostumbrados a entregar deberes físicamente no tienen problemas para cumplir lo dispuesto por el docente; mientras que el grupo experimental señala dificultades en el manejo de la plataforma virtual pese a que se les ha socializado adecuadamente; al principio ciertos estudiantes denotan problema para combinar la práctica virtual con lo recibido en clase.

Tabla 4: Segundo parcial

\begin{tabular}{llll}
\hline Segundo parcial & $\square$ & s & N \\
\hline Grupo Control & 6,52 & 1,92 & 47 \\
Grupo Experimental & 8,55 & 1,14 & 47 \\
\hline
\end{tabular}

Fuente: Plataforma de calificaciones OASIS

Elaborado por: Autores

La tabla 4 visualiza el promedio alcanzado por los estudiantes del nivel 1 en el segundo parcial. El grupo control tiene 6,52 $\pm 1,92$. Estos resultados demuestran que los estudiantes bajaron su rendimiento con respecto al primer parcial $(7,29)$. Sin embargo, también señala que existen estudiantes que alcanzaron una nota máxima de 8,44; así mismo quienes bajaron su rendimiento a 4,6 .

El grupo experimental en cambio alcanzó un promedio de 8,55 $\pm 1,14$ en 47 participantes. Estos resultados de segundo parcial claramente demuestran que los estudiantes ya mejoraron su destreza con la plataforma virtual educativa como herramienta didáctica que contribuye al mejor empleo del idioma puesto que el estudiante tiene la oportunidad de escuchar y practicar una pronunciación correcta, repasar las lecciones parciales y comprobar donde se equivocó, tareas de completación, escogimiento, organización de palabras, formación de oraciones, frases y diálogos que son evaluados inmediatamente.

Tabla 2: Tercer Parcial

\begin{tabular}{lcll}
\hline Tercer parcial & $\square$ & s & N \\
\hline Grupo Control & 5,44 & 2,35 & 47 \\
Grupo Experimental & 9,21 & 0,91 & 47 \\
\hline
\end{tabular}

Fuente: Plataforma de calificaciones OASIS

Elaborado por: Autores

La tabla 4 señala que el grupo control obtuvo un promedio de 5,44 \pm 2,35. Estos resultados demuestran el rendimiento bajo de los estudiantes que 
pueden deberse a varios motivos, entre ellos puede ser que los estudiantes demuestran más interés por las materias de su carrera y se descuidan de la materia de inglés.

El grupo experimental por su parte ha alcanzado un promedio de 9,21 $\pm 0,91$. Estos resultados son satisfactorios y demuestran que los participantes se encuentran motivados con el nuevo recurso didáctico utilizado, han captado mejor los conocimientos que les han permitido construir un aprendizaje significativo al momento de realizar las tareas y demás actividades vinculadas entre el texto y la plataforma virtual educativa.

Tabla 3: Promedio final

\begin{tabular}{llll}
\hline Tercer parcial & $\square$ & s & N \\
\hline Grupo Control & 6,42 & 2,35 & 47 \\
Grupo Experimental & 8,29 & 0,50 & 47 \\
\hline
\end{tabular}

Fuente: Plataforma de calificaciones OASIS

Elaborado por: Autores

El promedio final alcanzado por el grupo control es de 6,42 $\pm 2,35$ mientras que el grupo experimental es de 8,29 $\pm 0,50$. Estos resultados demuestran que la utilización las buenas prácticas docentes a través de un texto vinculado a una plataforma virtual educativa como herramienta didáctica, arrojó resultados positivos porque los estudiantes desarrollaron de mejor forma las habilidades comunicativas necesarias para el aprendizaje y dominio del idioma extranjero.

Tabla 4: Conocimiento previo y posterior Grupo control Grupo Experimental

\begin{tabular}{lllllll}
\hline Conocimiento & $\square$ & $\mathbf{S}$ & $\mathbf{N}$ & $\square$ & $\mathbf{s}$ & $\mathbf{N}$ \\
\hline Inicial & 4,22 & 1,86 & 47 & 3,37 & 1,64 & 47 \\
Final & 6,42 & 2,35 & 47 & 8,29 & 0,50 & 47 \\
\hline
\end{tabular}

La tabla 6 muestra los resultados obtenidos del análisis descriptivo del nivel de conocimientos previo y posterior de los grupos de control y experimental sobre el aprendizaje de inglés en el Nivel 1 de un Centro de Idiomas Universitario.

Los promedios en el grupo de control son de 4,22 $\pm 1,86$ y $6,42 \pm$ 2,35 para el conocimiento previo y posterior de los participantes que recibieron clases mediante el sílabo.

Los promedios para el grupo experimental es de 3,37 $\pm 1,64$ y 8,29 \pm 0,50 para el conocimiento previo y posterior respectivamente para los estudiantes que recibieron clases mediante un texto guía vinculado a una plataforma virtual educativa como herramienta didáctica y como instrumento de buena práctica docente.

El estudio inferencial de datos se estableció a partir de la comparación de medias con la aplicación de la Prueba T Student para analizar el nivel de 
conocimientos adquiridos entre los grupos de control y experimental al inicio del semestre, logrando determinar que el conocimiento inicial tanto en el grupo de control como experimental no difiere significativamente en la prueba de diagnóstico $(\mathrm{p}>0,05)$.

T student $\mathrm{p}>0,05$

$\mathrm{T}$ - independiente $\mathrm{p}<0,05$

Además, fue necesario desarrollar el análisis estadístico a fin de establecer si existe diferencia significativa en el conocimiento después, siendo preciso aplicar la prueba $T$ independiente la cual demuestra un valor de $p>0,05$ esto indica que existe una diferencia significativa entre el grupo de control y experimental.

\section{Conclusion}

El diagnóstico inicial permitió determinar que la mayoría de los estudiantes que ingresan al primer nivel de inglés presentan deficiencia en los conocimientos; tanto en el grupo de control como en el grupo experimental. Los resultados fueron comprobados con los obtenidos en los exámenes internacionales y estandarizados aplicados (TOEFL), donde se indica que los estudiantes del último año de Bachillerato General Unificado del Ecuador de los planteles educativos públicos se ubican en un nivel bajo. Este escenario permitió obtener una línea base para que el docente desarrolle las actividades contenidas en el sílabo de la asignatura, con la ayuda de las plataformas virtuales como buenas practicas docentes.

Los registros de calificaciones en las tareas presentaron novedades en un inicio, por cuanto los estudiantes del grupo de control, obtienen un mejor promedio que los estudiantes del grupo experimental, debido a que la entregar de tareas físicas no tuvieron dificultades de entrega; mientras que en el grupo experimental la ambientación del uso de la plataforma virtual presentó inconveniente para la entrega de las tareas dispuestas por el docente; por lo que se necesitó fortalecer a través de la guía docente el procedimiento. Este escenario fue superado y compensando con la realización de otras actividades interactivas, que en comparación al grupo de control fueron más dinámicas e incentivas; incentivando en los estudiantes en construir el conocimiento de forma autónoma y colectiva.

A medida que se desarrollaban las actividades con la plataforma virtual, se pudo observar que los estudiantes del grupo experimental mejoraban las calificaciones de las notas parciales acumulativas; es decir mejoraban su rendimiento académico en comparación con los estudiantes del grupo de control. La mejora del rendimiento se debió a que los estudiantes del grupo experimental, tenían a disposición recursos y actividades publicadas en la plataforma virtual para el aprendizaje del idioma inglés. Lo que le permitía al estudiante realizar retroalimentación de conocimiento a través de la 
repetición de ejercicios, comprobación de donde se equivocó, repaso de destrezas, etc.

De acuerdo a los resultados obtenidos se puede concluir que la utilización de buenas prácticas docente a través de un texto vinculado a una plataforma virtual educativa como herramienta didáctica para el aprendizaje de inglés en un Centro de Idiomas Universitario es positivo porque se los estudiantes alcanzaron promedios superiores en relación a la forma tradicional de impartir clases a través de un sílabo.

\section{References:}

1. Chaves, L. S. (2016). Interacción profesor-estudiante para el desarrollo de buenas prácticas docentes en el ámbito universitario. Portal Revistas Académicas. Núm.24. ISSN: 1659-1933, 8.

2. Fingermann, H. (2013). Buenas prácticas docentes. Obtenido de https://educacion.laguia2000.com/ensenanza/buenas-practicasdocentes

3. Gabaldón, E. (2011). Relación entre las buenas prácticas docentes y la satisfacción del estdiantes en el seguimiento de la calidad de las titulaciones. Obtenido de Universidad de Alicante: https://web.ua.es/es/ice/jornadas-redes/documentos/ponencias/evagabaldon.pdf

4. Heredia, V. (10 de 11 de 2017). El nivel de Inglés en el Ecuador todavía es bajo. Diario El Comercio, pág. 1.

5. Hernández, R. (2015). Metodología de la Investigación. México: MacGraw Hill.

6. Junta de Andalucía. (2012). Guía de Bueans Prácticas Docentes. Agencia Andaluza de Evaluación Educativa, 136.

7. Marqués, P. (2002). Buenas prácticas docentes. Obtenido de http://peremarques.pangea.org/bpracti.htm

8. Ortega, D. (2017). La Educación Ecuatoriana en Inglés: Nivel de dominio y competencias linguisticas de los estudiantes rurales. INDTECA. Instituto Internacional de Investigación y Desarrollo Tecnológico Educativo C. A. Vol 2. Núm. 6.

9. Osorio, I. (2017). Conocimientos, actitudes y prácticas docentes que favorecen la formación del carácter en estudiantes universitarios; una propuesta de evaluación. Red de Revistas Científicas de América Latina y el Caribe, España. UAEM REDALYC. DOI:http://dx.doi.org/10.6018/reifop.20.3.276171.

10. Rosenfeld, A., \& Fernández, M. (2012). Buenas prácticas en cooperación sur-sur triangular de Chile: criterios y metodología de selección de casos. Agencia de Cooperación Internacional de Chile. 
11. UNESCO. (2014). 15 Buenas prácticas docentes. Experiencias pedagógicas premiadas en el I Concurso Nacional de Buenas Prácticas Docentes. UNESCO.ORG, 83.

12. Vidal, M., \& Morales, I. (2009). Buenas prácticas docentes. Educación Media Superior v.23 n.1, 9.

13. VIU. (2015). Características y tipos deplataformas más utilizadas para estudiar a distancia. Obtenido de https://www.universidadviu.es/master-ciberseguridad/

14. Yáñez, L., \& Soria, K. (2017). Reflexión de Buenas Prácticas Docentes como eje de calidad en la Educación Universitaria: Caso Escuela de Ciencias Empresariales dela Universidad Católica del Norte. Formación Universitaria Vol. $10 \mathrm{~N}^{\circ} 5$. 